\title{
Furthering Precision in Sentinel Node Navigational Surgery for Oral Cancer: A Novel Triple Targeting System
}

Running Title: A Novel SN Navigation System for Oral SCC

Mr. Manish Chand MBA FRCS PhD, Department of Surgery and Interventional

Sciences, University College London Hospitals, NHS Trusts, GENIE Centre, University

College London, London, UK

Deborah S Keller MS MD, Department of Surgery and Interventional Sciences, University College London Hospitals, NHS Trusts, GENIE Centre, University College

London, London, UK

Laurence Devoto MBBS MSc MRCS, Department of Surgery and Interventional Sciences, University College London Hospitals, NHS Trusts, GENIE Centre, University College London, London, UK

Professor Mark McGurk, MD FRCS, Head and Neck Centre, University College London Hospitals, NHS Trusts, London, UK

The authors received no financial support for this work

Word Count: 799

Abstract Count: 238

Address correspondence to:

Mr. Manish Chand, MBA FRCS PhD

Consultant Colorectal Surgeon and Senior Lecturer

Department of Surgery and Interventional Sciences, University College London

Hospitals, NHS Trusts, GENIE Centre, University College London

235 Euston Rd, London, United Kingdom, NW1 2BU

Tel: 02034475879

Fax: 02034479218

manish.chand@uclh.nhs.uk 
Structured Abstract

Purpose: To describe an innovative sentinel lymph node (SLN) guidance approach using a radionuclide tracer, 3D augmented reality-guided imaging, and near infrared (NIR) fluorescence over-lay imaging with hand-held probes to optimize accuracy, efficiency, and precise navigation for sentinel node (SN) localization in head and neck cancer.

Methods: In a cT1N0M0 squamous cell carcinoma of the tongue, pre-operative radionuclide lymphoscintigraphy was performed with a sentinel node-specific radiolabeled tracer. Intraoperatively, a 3D hand-held augmented reality (AR) scanning SPECT probe assessed concordance of the SN with pre-operative SPECT-CT images. The real-time optical video was linked to the SPECT-CT images for added precision. Final guidance to the SN was performed using ICG fluorescence imaging.

Results: Dynamic and SPECT-CT showed bilateral lymphatic drainage from the tumor. The 3D hand-held AR SPECT probe SN localization was concordant with pre-operative imaging. The optical video successfully demonstrated the lymphatic drainage in realtime through a unique overlay fluorescence image. The ICG localized to the same nodes identified by both the SPECT-CT and hand-held SPECT images. The use of dual radiation and fluorescence tracers improved SN detection, especially for SN close to the injection site. The hand-held probes allowed the surgeon to dissect continuously, without needing to change tools.

Conclusions: The combination of augmented reality, nuclear medicine, and over-lay fluorescence imaging allowed greater accuracy for matching the preoperative imaging with intraoperative identification and precisely guiding the dissection. This method 
uniquely permitted the surgeon to efficiently dissect the $\mathrm{SN}$ with accurate visualization and optimal precision.

Keywords: oral squamous cell cancer; fluorescent imaging; sentinel lymph node; radioguided 3D imaging; augmented reality; precision surgery 
Short Communication

Accurately determining nodal status is paramount for appropriate cancer staging, adjuvant therapy, and survival. Oral squamous cell carcinoma (OSCC) is a prime example. With a 20-30\% incidence of lymph node metastases, the cervical lymph node status is the key prognostic factor $(1,2)$. To be risk adverse, elective neck dissections are commonly performed in clinically N0 patients, but have associated morbidity and risks, especially in pathologically N0 patients (3). Attention has been focused on sentinel node navigation surgery to improve diagnostic accuracy with lower risks.

Traditionally, intraoperative sentinel node $(\mathrm{SN})$ identification relied on acoustic signals generated by a $\gamma$-probe paired with sentinel node visualization using a visible dye $(4,5)$. Limitations in spatial resolution, sensitivity of the stain, and depth of the planes affect accuracy (6). Reports have discussed using near-infrared fluorescence imaging (FI) with Indocyanine Green (ICG), alone and paired with a radioactive tracer, to improve navigation through better spatial resolution, better accuracy, and real-time visualization of the $\mathrm{SN}$ within the surrounding anatomy (7-10).

Issues in signal penetration and diagnostic accuracy still persist, and another limitation is that the surgeon must activate the near-infrared (NIR) setting to visualize the node, losing focus on the anatomy and dissection plane. Thus, the surgeon currently cannot confirm the intraoperative field with the preoperative imaging or simultaneously continue their dissection while accurately visualizing nodes of interest in real-time. 
We report on an innovative sentinel lymph node (SLN) guidance approach using handheld radio-guided 3D imaging and near infrared (NIR) fluorescence imaging navigation probes. A non-sterile assistant may use this probe, allowing the surgeon to concentrate on the dissection. More importantly, it can overlay the fluorescence image onto the native anatomy. This combination of augmented reality, nuclear medicine, and fluorescence imaging allows precise anatomical localization, concordance between the preoperative imaging and intraoperative identification, efficiency for the surgical dissection with the hand-held probes, and greater precision guiding the oncologic dissection in real-time with augmented reality overlay.

A 71-year-old male with moderate-to-poorly differentiated left anterior tongue squamous cell carcinoma, staged as T1N0M0 by clinical examination, ultrasound and MRI was the subject.

Pre-operative radionuclide lymphoscintigraphy was performed with Tc99m-tilmanocept, a tracer that binds specifically to mannose binding receptors on the surface of resident dendritic cells and macrophages in the SLN. In the afternoon prior to surgery, $74 \mathrm{MBq}$ of the tracer was injected in four $0.1 \mathrm{ml}$ aliquots equidistant around the tumor. Dynamic and SPECT/CT scans show tracer uptake in SN bilaterally, inferring bilateral lymphatic drainage from the tumor (Fig 1).

The next morning, with the patient post-induction but preincision, augmented reality scanning with a 3D hand-held SPECT probe (SurgicEye®, Munich, Germany) showed complete concordance of the localization of $\mathrm{SN}$ in the bilateral neck with pre-operative SPECT/CT. The SurgicEye infrared navigation system registers the position of the 
patient's head through fiducial markers attached to the forehead and Gamma probe. As the probe is painted over the neck, the position of the gamma emitting foci (sentinel node) is pinpointed and appears as black spot on the real-time optical image of the patient. Localization security is achieved by computer registration of the SPECT/CT study with the real-time optical video of the patient during the surgery when the forehead fiducial marker is worn during SPECT/CT image acquisition (Fig 2). The technique enables precise anatomical localization of the target tissue. The SNB procedure in oral cancer is operator sensitive. Further security entails peri-tumoral administration of a colloid tracer bound with ICG, then using fluorescence imaging with a hand-held probe (SPY-PHI, Novadaq Technologies, Vancouver, BC, Canada). The colloid drained independently to the same set of nodes identified by both the SPECT/CT and hand-held SPECT images. By making a small incision in the region of highest gamma probe count, a non-sterile assistant could direct the hand-held fluorescent probe beam at the incision as the superficial layers were dissected to reveal an obviously fluorescent node (Figure 3). The image resolution was excellent and allowed precise dissection. The node and the lymphatic channel were immediately visible. Following excising the first node (Figure 3), a second pass was made with the both the gamma and hand-held fluorescent probes to ensure no additional nodal tissue. The use of a dual tracer (radiation and fluorescence) improved SN detection, particularly close to the injection site. Here, the blush of radiation around the injection site masks local nodes and makes the gamma probe uninformative. With this method, these SN's can be seen fluorescing in the surgical bed (Fig 4) and even after resection (Fig 5). 
This technology has potentially wide application at other tumor sites, especially if handheld SPECT proves reliable and can replace traditional SPECT/CT. SN's could be detected preoperatively and tumors that could formerly only be accessed by radical dissection under anesthesia would become candidates for SNB, potentially decreasing the morbidity and increasing the surgical precision in OSCC. 


\section{Compliance with Ethical Standards}

Mr. Chand is a consultant for Novadaq Technologies outside of the scope of this work. Mr. hand has received speaker and teaching honorarium from Novadaq Technologies unrelated to this work.

Dr, Keller declares that she has no conflict of interest.

Dr. Devoto declares that he has no conflict of interest.

Professor McGurk declares that he has no conflict of interest.

Ethics Approval and consent to participate

This work involves no experimental devices for human participants.

Informed consent was obtained from all individual participants included in the study. All procedures performed in studies involving human participants were in accordance with the ethical standards of the institutional and/or national research committee and with the 1964 Helsinki declaration and its later amendments or comparable ethical standards. This article does not contain any studies with animals performed by any of the authors.

\section{Competing Interests}

Mr. Chand, Dr. Keller, Dr. Devoto, and Professor McGurk have no competing interests

\section{Funding}

There was no funding or financial support for this work

Consent for Publication

Consent for publication was obtained from the individual to cover use of any data; this is available upon request.

\section{Authors' Contribution}

Mr Chand made substantial contributions to conception and design, acquisition of data, was involved in drafting the manuscript and revising it critically for important intellectual content, gave final approval of the version to be published, and agreed to be accountable for all aspects of the work in ensuring that questions related to the accuracy or integrity of any part of the work are appropriately investigated and resolved.

Dr, Keller made substantial contributions to conception and design, acquisition of data, was involved in drafting the manuscript and revising it critically for important intellectual content, and gave final approval of the version to be published.

Dr.Devoto made substantial contributions to conception and design, acquisition of data, was involved in drafting the manuscript and revising it critically for important intellectual content, and gave final approval of the version to be published.

Professor McGurk made substantial contributions to conception and design, acquisition of data, was involved in drafting the manuscript and revising it critically for important intellectual content, gave final approval of the version to be published, and agreed to be accountable for all aspects of the work in ensuring that questions related to the accuracy or integrity of any part of the work are appropriately investigated and resolved. 


\section{References}

1. Broglie MA, Stoeckli SJ. Relevance of sentinel node procedures in head and neck squamous cell carcinoma. Q J Nucl Med Mol Imaging. 2011;55:509-520.

2. Coughlin A, Resto VA. Oral cavity squamous cell carcinoma and the clinically n0 neck: the past, present, and future of sentinel lymph node biopsy. Curr Oncol Rep. 2010;12:129-135.

3. Murase R, Tanaka H, Hamakawa T et al. Double sentinel lymph node mapping with indocyanine green and 99m-technetium-tin colloid in oral squamous cell carcinoma. Int J Oral Maxillofac Surg. 2015;44:1212-1217.

4. Nieweg OE, Jansen L, Kroon BB. Technique of lymphatic mapping and sentinel node biopsy for melanoma. Eur J Surg Oncol. 1998;24:520-524.

5. Kapteijn BA, Nieweg OE, Liem I et al. Localizing the sentinel node in cutaneous melanoma: gamma probe detection versus blue dye. Ann Surg Oncol. 1997;4:156160.

6. Brouwer OR, Buckle T, Bunschoten A et al. Image navigation as a means to expand the boundaries of fluorescence-guided surgery. Phys Med Biol. 2012;57:3123-3136.

7. van den Berg NS, Brouwer OR, Klop WM et al. Concomitant radio- and fluorescence-guided sentinel lymph node biopsy in squamous cell carcinoma of the oral cavity using ICG-(99m)Tc-nanocolloid. Eur J Nucl Med Mol Imaging. 2012;39:1128-1136.

8. Hayashi T, Furukawa H, Oyama A et al. Sentinel lymph node biopsy using realtime fluorescence navigation with indocyanine green in cutaneous head and neck/lip mucosa melanomas. Head Neck. 2012;34:758-761.

9. Nakamura Y, Fujisawa Y, Nakamura Y et al. Improvement of the sentinel lymph node detection rate of cervical sentinel lymph node biopsy using real-time fluorescence navigation with indocyanine green in head and neck skin cancer. J Dermatol. 2013;40:453-457.

10. Brouwer OR, Buckle T, Vermeeren L et al. Comparing the hybrid fluorescentradioactive tracer indocyanine green-99mTc-nanocolloid with 99mTc-nanocolloid for sentinel node identification: a validation study using lymphoscintigraphy and SPECT/CT. J Nucl Med. 2012;53:1034-1040.

Figure Diagram

- Figure 1 Dynamic and SPECT-CT scans showing uptake of tracer in sentinel nodes bilaterally

- Figure 2 Forehead fiducial marker worn during the acquisition of the SPECT/CT images, which allows computer registration of the SPECT/CT study with the realtime optical video of the patient during the surgery

- Figure 3 Assistant directing the beam of the hand-held fluorescent probe

- Figure 4 Lymph Node Bed

- Figure 5 Flourescing Lymph Nodes After Resection 